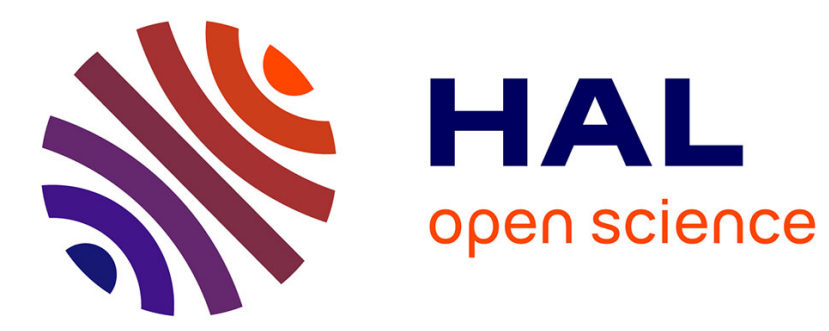

\title{
Les consommateurs perçoivent-ils le prix des produits du commerce équitable comme étant juste?
}

Sihem Dekhili, Mantiaba Coulibaly, Ivan Dufeu

\section{To cite this version:}

Sihem Dekhili, Mantiaba Coulibaly, Ivan Dufeu. Les consommateurs perçoivent-ils le prix des produits du commerce équitable comme étant juste?. Décisions Marketing, 2017, 85, pp.83-100. 10.7193/DM.085.83.100 . hal-02794183

\section{HAL Id: hal-02794183 \\ https://univ-angers.hal.science/hal-02794183}

Submitted on 25 Jun 2021

HAL is a multi-disciplinary open access archive for the deposit and dissemination of scientific research documents, whether they are published or not. The documents may come from teaching and research institutions in France or abroad, or from public or private research centers.
L'archive ouverte pluridisciplinaire HAL, est destinée au dépôt et à la diffusion de documents scientifiques de niveau recherche, publiés ou non, émanant des établissements d'enseignement et de recherche français ou étrangers, des laboratoires publics ou privés. 


\title{
Les consommateurs perçoivent-ils le prix des produits du commerce équitable comme étant juste?
}

\author{
Sihem Dekhili", Mantiaba Coulibaly"* et Ivan Dufeu*** \\ * Laboratoire HuManiS (EA 7308), École de Management Strasbourg - Université de Strasbourg \\ ** Laboratoire GRM (EA 4711), Université Côte d'Azur \\ ${ }^{* * *}$ Laboratoire LEMNA (EA 4272), ONIRIS Nantes/ LARGECIA
}

\begin{abstract}
Résumé
Le principe de prix juste constitue l'un des piliers du commerce équitable (CE) depuis ses origines. Dans la littérature, cette question est généralement abordée sous l'angle des producteurs, avec le regard de l'analyste ou du chercheur : sont-ils rémunérés correctement pour leur travail ? La présente recherche, qui s'appuie sur des entretiens menés auprès de consommateurs français, se propose d'explorer la justice liée au prix en se plaçant de leur point de vue : le prix qu'ils paient pour les produits issus du CE leur semble-t-il juste ? En nous appuyant sur la théorie de l'équité, nous avons observé que les individus, dans leur perception de la justice liée au prix, ne mobilisent pas de mécanisme comparatif. Ils adoptent souvent des discours qui relèvent des principes de la justice distributive au sens d'Aristote en s'intéressant notamment à la répartition des prix et des marges dans les filières de CE. Dans ce contexte, la perception de la justice liée au prix est conditionnée par la confiance accordée aux organisations intervenant dans ces filières.
\end{abstract}

Mots-clés : confiance, perception des consommateurs, prix juste, produits équitables, théorie de l'équité.

\begin{abstract}
Do consumers perceive the price of fair trade products as fair?

The principle of fair price has been a cornerstone of fair trade (FT) since its origins. In the literature, this question is generally studied from the perspective of producers, via the researcher's point of view: are they correctly paid for their work? This article, which is based on interviews with French consumers, explores price fairness from the consumers' point of view: is the price paid for FT products considered fair?

Based on the theory of fairness, we observe that individuals, in their perception of fairness related to price, do not mobilize a comparative mechanism. They often adopt a discourse that is based on the principles of distributive justice according to Aristote with an emphasis on the price and margin distribution in the FT product chains. In this context, the perception of fairness related to the price is conditioned by the confidence level given to the organizations operating in these chains.
\end{abstract}

Key words: confidence, consumers' perception, fair price, fair products, theory of fairness.

Pour contacter les auteurs : sihem.dekhili@unistra.fr, mantiaba.coulibaly@unice.fr et ivan.dufeu@oniris-nantes.fr

DOI : 10.7193/DM.085.83.100 - URL : http ://dx.doi.org/10.7193/DM.085.83.100

Dekhili S., Coulibaly M. et Dufeu I. (2017), Les consommateurs perçoivent-ils le prix des produits du commerce équitable comme étant juste?, Décisions Marketing, 85, 83-100. 


\section{Introduction}

Le cadre législatif du commerce équitable (CE) en France a changé depuis l'adoption à l'Assemblée nationale de la nouvelle Loi n $2014-856$ du 31 juillet 2014 relative à l'économie sociale et solidaire, qui modifie la Loi $n^{\circ}$ 2005-882 du 2 août 2005. Dans son article 94, cette nouvelle loi introduit explicitement l'obligation de pratiquer des prix « justes », reposant sur : " le paiement par l'acheteur d'un prix rémunérateur pour les travailleurs, établi sur la base d'une identification des coûts de production et d'une négociation équilibrée entre les parties au contrat $»^{1}$. Dans un contexte où les notions de «prix juste » ou de « prix équitable » (ces deux concepts traduisant le «fair» en anglais) font l'objet d'une grande diversité d'interprétations (Diaz-Pedregal, 2007), cette formulation officielle est porteuse de sens. La loi mentionne en effet deux fondements de la fixation des prix dans le $\mathrm{CE}$ : le prix payé par le premier acheteur aux petits producteurs doit permettre à ces derniers de vivre dignement de leur production, et il ne doit pas être fixé dans le cadre d'un rapport de force entre offre et demande (défavorable généralement aux petits producteurs). Elle renvoie à l'idée de « bonne économie » chez Aristote, et plus précisément au concept de justice commutative, c'est-à-dire à l'idée de donner et recevoir ce qui est juste dans l'échange entre deux personnes égales (Aristote, 1998). Les acteurs du commerce équitable ont développé divers outils et méthodologies de fixation de " prix justes » pour le producteur en amont de la filière, afin de traduire cet engagement (publication de prix minimum garantis, versement de primes pour le développement, etc.). Ces principes varient quelque peu selon les organisations de $\mathrm{CE}$, renvoyant à leur interprétation de la notion de justice liée au prix (Le Velly, 2008).

1/ http://www.legifrance.gouv.fr/affichTexte.do;j sessionid $=8$ D1374D65CFCD204401D39339D206 3A3.tpdjo12v_1?cidTexte=JORFTEXT0000293132 96\& dateTexte $=20140801$
Dans tous les cas, le principe qui sous-tend le CE est qu'il faut a minima rétablir un prix juste pour le producteur en amont de la filière. À l'autre bout de la chaîne, le constat est que le consommateur doit quant à lui généralement débourser davantage pour les produits équitables, vendus en moyenne 10 à $20 \%$ plus cher que leurs équivalents conventionnels (Artisans du Monde, 2007). Peut-on supposer que le consommateur fait le lien entre le principe et le constat ? Que s'il adhère au principe, alors il considérera comme juste le prix qu'il paye pour le produit fini ?

Cette question est un enjeu majeur pour le CE, car le prix des produits issus de ces filières s'avère être un frein majeur à l'acte d'achat. Dans nombre d'enquêtes conduites auprès des consommateurs, le premier argument avancé pour expliqué le «non-achat» de produits du CE est en effet leur prix ${ }^{2}$. Dans ce contexte, en dépit des attitudes positives déclarées, les achats de produits éthiques en général, et équitables en particulier, restent limités. Une étude réalisée par TNS Sofres et l'Observatoire Cetelem en 2013 montrait par exemple que, si $53 \%$ des Français indiquaient qu'ils tenaient de plus en plus compte des dimensions éthiques dans leurs choix de consommation, leur panier moyen en produits du CE se situait autour de 6,5 euros par $a^{3}$. La littérature académique propose certes, en dehors du prix élevé des produits du CE (De Pelsmacker et alii, 2005), de nombreuses explications à cet écart entre attitude et comportement (attitude behavior gap) : scepticisme et manque d'information des consommateurs (Pernin et Petitpretre, 2012 ; Castaldo et alii, 2009), manque d'appropriation (Gurviez et Sirieix, 2013) ou manque de disponibilité des produits (Carrigan et Attala, 2001). Mais le fait de réduire ces barrières, en améliorant par exemple l'information au

2/ TNS SOFRES (2010), Les Français et le commerce équitable, sondage effectué pour INC 60 millions de consommateurs. Paris.

3/ Selon une étude publiée par la PFCE en 2014 (Les chiffres clés du commerce équitable en France). 
consommateur (Gurviez et Sirieix, 2013), en diffusant plus largement les produits ou en renforçant les procédures de certification et la transparence (Balineau et Dufeu, 2012), n'a pas fait disparaître cet écart entre attitude et comportement.

Dans ce contexte, l'objectif de la présente recherche est de tenter d'en savoir plus sur la manière dont les consommateurs perçoivent le prix des produits du CE, notamment au regard du critère de justice. Dans la littérature académique, les recherches qui se sont plus particulièrement intéressées à l'équité ou à la justice dans la relation d'échange entre partenaires du CE ont surtout envisagé la question de la rémunération des producteurs (Béji-Becheur, Diaz-Pedregal et Özçağlar-Toulouse, 2008 ; Galtier et Diaz Pedregal, 2010 ; Le Velly, 2008). Les travaux en marketing qui ont exploré le prix payé par le consommateur final, plus proches de notre questionnement, étaient surtout centrés sur la question du consentement à payer (De Pelsmacker et alii, 2005 ; Dufeu et alii, 2014). Mais si ces travaux sont utiles pour fournir des indicateurs de la valorisation potentielle d'un produit (généralement d'un label) équitable, ils ne nous permettent pas de comprendre ce qui conduit les consommateurs à considérer que tel prix d'un produit du $\mathrm{CE}$ est juste ou injuste.

Cette interrogation a donc guidé notre recherche exploratoire : sur quels principes les consommateurs de produits équitables s'appuient-ils pour déterminer si le prix qu'ils payent pour les produits du CE est un prix juste ou injuste ? Quels sont les éléments d'information qu'ils mobilisent pour se faire un avis ?

Dans cet article, nous présentons tout d'abord les spécificités du prix telles que définies par les organisations du CE avant d'explorer leur perception par le consommateur. Nous nous appuyons notamment sur la théorie de l'équité, laquelle est éclairante non seulement sur les critères d'appréciation de la justice/injus- tice liée au prix, mais aussi sur les indicateurs mobilisables par les consommateurs pour se faire un avis au cas par cas. Nous présentons ensuite la méthodologie et les résultats essentiels de l'étude. Nous soulignons enfin les principales implications managériales et limites de cette recherche.

\section{Le prix équitable dans le CE}

Le commerce équitable vise originellement à répondre à l'injustice de prix auquel sont confrontés certains petits producteurs notamment dans le cadre du commerce conventionnel. Cette injustice vient de ce que ce dernier ne permet pas toujours de valoriser certaines caractéristiques extrinsèques de biens produits par des petits producteurs des pays en développement.

\section{I.I. Mettre fin au prix inéquitable pour le petit producteur dans le commerce conventionnel}

Sur certains marchés de produits primaires, le prix résultant de la confrontation entre l'offre et la demande peut être si faible qu'il ne couvre pas les coûts de production de l'offreur. Ceci se produit souvent dans les filières alimentaires où des petits paysans nombreux et dispersés font face à des acheteurs plus puissants. L'excès d'offre n'est pas seul en cause dans l'établissement d'un prix faible : la domination de la demande peut aussi venir d'une situation de monopsone ou d'oligopsone local, et/ou d'une asymétrie d'information favorable aux acheteurs (le fameux cas des « coyotes » en Amérique Latine ${ }^{4}$ ). Il n'y a pas réellement de libre marché car les offreurs ne sont pas libres de refuser un prix (étant contraints de vendre pour se nourrir) alors que les acheteurs le sont.

4/ Terme utilisé au Mexique pour décrire les intermédiaires locaux qui exerçaient leur pouvoir de négociation au détriment des petits producteurs de café. 
Encadré 1 : La détermination du prix juste par les organisations du CE

\section{Le système Fairtrade/Max Havelaar}

Selon FLO (Fairtrade Labelling Organization)/Max Havelaar, « un prix juste dans un contexte local ou régional est convenu après dialogue et concertation. Cela couvre non seulement les coûts de production mais permet également une production qui est socialement juste et respectueuse envers l'environnement. Il fournit un prix juste aux producteurs et prend en compte le principe de salaire égal pour un travail équivalent entre hommes et femmes. Les organisations de Commerce Équitable assurent un paiement immédiat à leurs partenaires et, dès que cela est possible, des préfinancements avant la récolte ou la production » (PFCE, 2010).

Le prix équitable se décompose en deux parties :

- un prix minimum garanti (prix minimum du CE) établi pour couvrir les coûts de production durable et assurer les besoins élémentaires de la famille des producteurs. Il est fixé pour chaque matière première, dans le cadre du Comité des standards et du Conseil d'Administration (Le Velly, 2008). Le prix minimum du CE est le prix le plus bas possible qu'un acheteur peut payer à des producteurs, pour être certifié selon les standards du CE ;

- une prime de développement (prime du CE) établie pour doter les organisations de producteurs d'une capacité d'autofinancement et d'investissement de base, tant dans le domaine social (dispensaires, scolarisation...) que dans celui de la production (matériel de séchage) (PFCE, 2010).

\section{Le système WFTO (World Fair Trade Organisation)}

WFTO souligne également le caractère central de la question de la justice liée aux prix et aux salaires. Selon l'organisation, un salaire juste permet d'éviter une escalade de prix, d'améliorer l'efficacité et la productivité, et de permettre une transparence des coûts et des prix. La méthode est également fondée sur des analyses contextuelles, prenant en compte les contingences locales et les besoins actuels des artisans, mais il n'existe pas de politique de prix centralisée, telle que celle prévalant dans le système Fairtrade. Les différences résident notamment dans la responsabilité donnée aux différents acteurs de la filière en aval des petits producteurs, notamment aux acheteurs ; ils doivent renforcer leurs capacités et s'engager sur des commandes régulières, contrôler les coûts internes, accroître leur efficience, identifier le courant dominant sur le marché, minimiser la flambée des prix de la TVA, etc. (WFTO, 2013).

Dans ce contexte, le prix peut être inéquitable pour les petits producteurs, pour deux raisons : parce qu'il ne permet pas de rémunérer correctement le travail fait par celui-ci ; parce qu'il est établi dans le cadre d'une relation dominant-dominé. Le CE vise notamment à rétablir une forme de justice en jouant sur ces deux tableaux, comme le rappelle la loi du 31 juillet 2014, et comme le précise le consensus FINE 5 (1999).

5/ Regroupement informel des quatre principaux réseaux (à l'époque) internationaux de commerce équitable : Fair Trade Labelling Organisations (FLO), International Fair Trade Association (IFTA, devenue World Fair Trade Organisation ou WFTO), Network of European Worldshops (NEWS), European Fair Trade Association (EFTA). Consensus FINE accessible à http://www.fair-tradehub.com/support-files/fair-trade-definition.pdf
La détermination du prix équitable repose, d'une part, sur une volonté d'objectiver la valeur de l'offre par estimation des coûts de production et des coûts associés aux exigences spécifiques du CE (notamment sociales et environnementales) ; dans le système Fairtrade Int (Encadré 1), cette volonté a conduit à l'élaboration des tableaux du prix minimum du CE et de la prime du CE par produit. La détermination du prix se fait, d'autre part, dans le cadre de négociations se voulant équilibrées entre les parties : producteurs, acteurs commerciaux et représentants des organisations du CE (Le Velly, 2008). 


\subsection{Valoriser l'ensemble des dimensions de la valeur}

L'idée de prix juste est formulée ainsi par Thomas d'Aquin (1273), reprenant l'idée de justice commutative chez Aristote ${ }^{6}:$ "Si le prix excède la valeur d'une chose ou inversement, l'égalité requise par la justice est manquante » (cité par Robert-Demontrond, 2008, p. 221). Dans la philosophie scolastique en effet, l'idée centrale est qu'aucun des partenaires ne doit profiter de l'échange au détriment des autres. Par exemple, un commerçant vend un produit à un prix correspondant à sa valeur additionnée d'une marge lui permettant de vivre de son activité commerciale. Cela pose naturellement la question essentielle de la valeur « réelle » du bien, la question centrale des théories économiques classiques et néoclassiques qu'il serait très présomptueux de vouloir trancher ici. Il importe simplement de rappeler que le CE s'intéresse à des biens dont le prix de marché ne reflète pas l'ensemble des dimensions de valeur pertinentes. Un paquet de café, par exemple, a une valeur d'usage personnel (qualités organoleptiques et sanitaires) et une valeur non liée à l'usage personnel, mais à certaines de ses qualités extrinsèques (Robert-Demontrond, 2008). En l'absence de mécanismes spécifiques de révélation des informations, des qualités extrinsèques comme les conditions sociales ou environnementales de la production ne sont pas intégrées dans le prix de marché. Le principe du CE est de valoriser ces caractéristiques extrinsèques

6/ Aristote (1998) distingue justice distributive et justice commutative. La justice commutative ne se préoccupe pas de la contribution respective des personnes, elle établit une équivalence entre choses et choses. La justice commutative pense l'égalité comme l'égalité arithmétique, ignore les différences entre les individus et donne à chacun la même part. $\mathrm{Au}$ contraire, « la justice distributive repose sur une égalité non absolue, mais proportionnelle. Par exemple, l'attribution des charges et des honneurs dans la cité devra se faire selon la proportion des mérites et des apports personnels de chaque citoyen ». (http://www.universalis.fr/encyclopedie/ justice-distributive/). additionnelles par rapport à un produit conventionnel (Béji-Becheur, Diaz-Pedregal et Özçağlar-Toulouse, 2008).

Les produits du CE sont donc supposés porter des promesses de valeurs différentes (valeur non liée à l'usage personnel) et additionnelles aux consommateurs. Dans le CE, la « subvention » versée à une pratique environnementale, sociale et/ou économique plus vertueuse est volontaire, donc subordonnée aux préférences individuelles. Il est possible, dans ce contexte, de faire l'hypothèse que le consommateur sera disposé à payer un surprix à condition qu'il le considère comme juste, selon ses propres critères et indicateurs, au regard des qualités intrinsèques et extrinsèques qu'il perçoit.

Il importe donc pour les acteurs du CE de comprendre ce qui peut conduire les consommateurs à ressentir un sentiment d'injustice/ de justice face au prix d'un produit du CE.

\section{Un prix juste pour les consommateurs dans le CE ?}

Les produits équitables sont généralement vendus plus chers que leurs équivalents conventionnels. Selon Robert-Demontrond et Özçağlar-Toulouse (2011), en acceptant de payer ce surprix, les consommateurs réduisent volontairement leur propre niveau de vie pour accroître celui des producteurs ( $\mathrm{em}$ powerment des petits producteurs). Toutefois, le fait que le petit producteur en amont soit mieux rémunéré peut être une condition nécessaire mais non suffisante pour que le prix final payé par le consommateur soit juste à ses yeux (Galtier et Pedegral 2010), et ce pour notamment deux raisons :

- d'abord parce que le prix final rémunère nombre d'autres acteurs de la filière (ne serait-ce que l'organisation du $\mathrm{CE}$ ), la partie revenant au petit producteur étant souvent très minoritaire (d'après Griffiths (2012), 1 à $18 \%$ du prix payé par le consommateur 
final revient au producteur en amont de la filière) ; le prix payé au petit producteur peut être juste, mais des intermédiaires, le distributeur par exemple, peuvent pratiquer des marges importantes et proposer un prix final injuste aux yeux du consommateur ;

- ensuite parce que les consommateurs sont, comme les producteurs, tous différents ; un consommateur peut se trouver lui aussi dans une situation économique défavorable pouvant rendre le surprix à payer pour un produit du CE difficile à supporter économiquement. S'il est juste de soutenir un petit producteur en difficulté, est-il juste de demander à un consommateur en difficulté de le faire ? S'il y a discrimination positive en amont, pourquoi pas en aval ?

En d'autres termes, les individus portent en permanence un jugement sur la répartition de la richesse dans les filières de production. Qui mérite de recevoir/payer quoi ? Tout cela renvoie non seulement à l'idée de justice commutative chez Aristote, à toutes les étapes de la filière, mais aussi à l'idée de justice distributive, qui porte sur la répartition des richesses entre individus. L'exigence de justice distributive se matérialise encore davantage dans la sphère du CE, l'une des qualités extrinsèques supposées des biens du CE étant que leur prix a conduit à rémunérer « justement » tous les acteurs de la filière (surtout dans le cas de WFTO).

\section{I. Les indicateurs de la justice liée au prix pour le consommateur : les apports de la théorie de l'équité}

Une littérature importante a traité la question de l'équité dans une relation d'échange et a été fondée en grande partie sur la logique de distribution coûts-profits entre les différentes parties. Elle fournit certaines clés de lecture concernant les indicateurs qui sont mobilisables par les consommateurs pour apprécier la justice/injustice liée au prix qu'ils paient.

La théorie de la justice distributive (Homans, 1961), la théorie de l'équité (Adams, 1965) ou encore la théorie des comparaisons sociales (Martins et Monroe, 1994) ont été focalisées sur le résultat final de l'échange et analysent la façon dont les bénéfices sont partagés et les avantages et les charges sont divisés entre les parties (Homans, 1961).

Reste que le consommateur doit se faire un avis sur cette répartition, en fonction de ses propres convictions et de sa conception personnelle de la justice, mais aussi en fonction des informations dont il dispose. Pour ce faire, trois mécanismes principaux sont identifiés par la littérature : comparaison, justification, degré de confiance.

\section{Comparaison}

Selon Homans (1961) ou Adams (1965), le jugement de la justice liée au prix est le résultat d'une estimation individuelle qui convoque un mécanisme de comparaison entre un prix de vente et un autre prix (considéré comme une norme ou une référence). Il s'agit de déterminer dans quelle mesure la différence entre les deux prix est raisonnable, acceptable ou justifiable (Xia, Monroe et Cox, 2004). Le jugement de la justice liée au prix est basé sur des transactions comparables qui impliquent des parties différentes. Le contraste entre les deux transactions peut expliquer la différence de prix. Xia, Monroe et Cox (2004) supposent que les différences de produits dans deux transactions distinctes, en réduisant fortement le degré de similarité entre les transactions, affaiblissent la perception de l'injustice liée au prix chez le consommateur.

\section{Justification}

La structure des coûts et la capacité des vendeurs à les contrôler peuvent avoir un effet sur l'acceptabilité et l'équité perçue (Campbell, 2007). En analysant l'équité des résultats de négociations des prix, Maxwell, Nye et Maxwell (1999) introduisent une distinction entre le prix préféré (c'est-à-dire ce que l'on veut) et le prix raisonnable (c'est-à-dire ce que l'on croit être juste). Selon la logique 
de distribution coûts-profits, la perception d'un prix injuste provient notamment d'un manque de justification ou de la nature de la justification avancée dans le cas d'une augmentation de prix. Lorsque le consommateur estime que les vendeurs ont augmenté leurs prix dans l'objectif de profiter d'un accroissement de la demande ou d'une pénurie d'offre, sans aucune augmentation correspondante en termes de coûts, il perçoit alors ces nouveaux prix plus élevés comme injustes (Xia, Monroe et Cox, 2004).

\section{Confiance envers le vendeur}

Un autre facteur qui influence la perception de l'injustice liée au prix est la référence des consommateurs à leurs expériences antérieures. Dans ce contexte, la confiance envers le vendeur joue un rôle important (Ganesan, 1994). Lorsque la relation vendeur-acheteur est bien établie, un prix affiché équivalent ou inférieur au prix attendu est considéré par le consommateur comme le bénéfice de sa relation privilégiée avec le vendeur. En revanche, un prix affiché supérieur peut conduire, soit à une perception d'injustice liée au prix plus importante, soit à la recherche par le consommateur de raisons légitimes pour expliquer l'augmentation de prix. De plus, les consommateurs peuvent faire référence à leurs propres connaissances (référence au prix de marché par exemple) et croyances sur les pratiques des vendeurs (tactiques de fixation des prix) afin d'ajuster leurs évaluations sur la justice liée au prix (Xia, Monroe et Cox, 2004).

\subsection{Les conséquences négatives des perceptions d'une injustice liée au prix}

La perception d'une injustice liée au prix induit, d'une part, une baisse de la valeur perçue des produits qui résulte d'une perception d'un sacrifice financier et, d'autre part, un accroissement de la sensibilité au prix (Martins et Monroe, 1994). Elle engendre chez le consommateur des réactions émotionnelles et comportementales négatives. Certaines émo- tions suscitent des actions qui peuvent avoir des conséquences négatives sur le vendeur, comme une moindre intention d'achat et l'apparition de plaintes (Olivier et Swan, 1989). Le consommateur, à travers ces actions, tente de s'auto-protéger contre d'éventuels inconvénients futurs ou de se venger. Il peut éventuellement rompre sa relation avec le vendeur pour aller chez son concurrent direct même si ce choix peut s'avérer peu profitable et irrationnel pour lui.

C'est notamment en s'appuyant sur ces enseignements de la théorie de l'équité que cet article vise donc à comprendre les perceptions des prix des produits équitables et à cerner leur niveau de justice du point de vue des consommateurs (Encadré 2).

\section{Les déterminants de la perception de la justice liée au prix : le cas du café équitable}

Les verbatim sont analysés à l'aune des enseignements de la théorie de l'équité. Les références utilisées par les personnes interrogées pour apprécier la justice liée au prix peuvent être regroupées en quatre thèmes : 1) la comparaison de prix, souvent mobilisée par les acheteurs selon la théorie de l'équité, est présente à la marge dans les discours des répondants ; 2) la justification du prix l'est en revanche davantage, se référant aux qualités intrinsèques et extrinsèques supposées du produit ; 3) une analyse de la distribution coûts/profits est également souvent abordée ; en lien avec ce dernier point, les appréciations s'appuient enfin sur 4) la confiance (relative) qu'ils ont dans les acteurs du CE.

\section{I. Un écart monétaire acceptable : faible similarité avec les autres produits et « fragilité » du prix de référence}

Selon les consommateurs, le prix contribue à la définition même d'un "produit équitable » : "pour moi, c'est un produit qui respecte un certain nombre de critères sur 
Encadré 2 : Détails de la méthodologie d'investigation du terrain

Les recherches étant peu nombreuses sur la compréhension de la perception du prix des produits du CE et sur la justice liée au prix du point de vue des consommateurs, une étude qualitative a été conduite. Il s'agit d'une enquête qui a porté sur un échantillon de consommateurs français de café. Le café a été choisi car il est considéré comme le produit le plus concerné par la différenciation basée sur la préoccupation sociale et environnementale. Il est aussi l'un des produits les plus consommés dans la catégorie des produits issus du commerce équitable (Max Havelaar, 2007).

Quarante-trois entretiens individuels en face à face ont été réalisés entre mai et octobre 2013. Les consommateurs ont été interrogés dans la rue, à la sortie de boutiques spécialisées de commerce équitable, à côté de quelques GMS, etc. La sélection des répondants s'est poursuivie jusqu'à saturation sémantique. Aucune incitation financière n'a été proposée.

Afin d'obtenir les entretiens les plus riches possibles, nous avons veillé à ce que l'échantillon des consommateurs interrogés (Annexe 1) soit le plus diversifié possible, en variant l'âge, le sexe et la catégorie socio-professionnelle. En outre, nous avons considéré des profils différents en termes de consommation des produits équitables (ancienneté et fréquence de consommation, lieux d'achat). Profitant d'une facilité d'accès à deux terrains par les auteurs, les entretiens ont été réalisés dans deux villes françaises : Strasbourg $(n=23)$ et Nice $(n=20)$.

Lors de nos enquêtes, nous avons mobilisé un guide d'entretien (Annexe 2), construit notamment en nous appuyant sur la revue de littérature. Il aborde successivement l'univers des produits équitables, l'évaluation de l'information que possèdent les répondants autour de ces produits, leur confiance envers la certification, la perception du prix du café équitable et de son niveau de justice, et enfin les habitudes de consommation de produits équitables.

Les entretiens ont duré 25 minutes en moyenne et ont été enregistrés puis intégralement retranscrits sur 200 pages environ. Une analyse de contenu thématique des verbatim recueillis a été réalisée de manière individuelle par deux des auteurs de cet article, en mobilisant le logiciel Nvivo8, un outil d'aide à l'analyse de discours. Puis les deux analyses ont été comparées et ont révélé une grande homogénéité des résultats (Kappa de Cohen $=0.82$ ). Au vu des similitudes dans les réponses des répondants strasbourgeois et niçois, l'analyse de contenu a été effectuée sur l'ensemble du discours recueilli et sans distinction selon le lieu de collecte des données. La structure de la grille d'analyse a été construite sur la base des thèmes relevés dans la littérature sur les déterminants de la perception de la justice liée au prix (comparaison, justification, confiance), et a été amendée à chaque lecture.

le prix » (Nadia, 26 ans). Globalement, les interviewés pensent qu'il s'agit d'un produit qui est vendu à un prix « correct » et «honnête » et qui est juste également pour eux, consommateurs : «c'est un produit qu'on a payé au juste prix» (Muriel, 61 ans), «le prix est équitable » (Myriam, 36 ans).

Toutefois, beaucoup avancent qu'il est difficile voire non pertinent de comparer avec d'autres produits : " je n'ai pas vraiment comparé le prix du café équitable avec le prix d'un autre café classique » (Roseline, 53 ans), «il [le café équitable] est un petit peu plus cher mais je pense que les cours $d u$ café étant mondiaux, ceux du commerce général ne sont peut-être pas justes » (Janine, 65 ans), au sens où ils ne reflètent pas réellement les coûts de production. Ceci favorise l'acceptation d'un prix élevé : «de toute façon qu'il soit plus élevé ne me gêne pas beaucoup parce que je sais que derrière il y a des choses qui sont mises en place» (Antoinette, 68 ans), "si on y croit on le paye. Tout à l'heure il y a un monsieur qui est venu qui a dit qu'il était au chômage et qui a pris 5 paquets de café Pérou, c'est le café le plus cher, alors quand on y croit on le paye (Giselle, 67 ans).

De plus, le prix dans le cas des produits équitables semble peu compatible avec l'idée d'un prix bas et à la recherche de bonnes affaires par le consommateur: « un produit ordinaire, il est payé très peu, on essaye de gratter pour payer le moins cher possible alors que pour les produits équitables nous voulons payer le juste prix » (Corinne, 57 ans), «c'est un prix 
acceptable, donc je ne le discute pas moi le prix $\gg$ (Antoinette, 68 ans).

$\mathrm{Au}$ total, nombre de répondants déclarent donc ne pas mobiliser réellement le mécanisme comparatif. Les différences entre produits dans deux transactions distinctes réduisent fortement le degré de similarité entre les transactions en question, et affaiblissent par conséquent la perception de l'injustice liée au prix chez le consommateur (Xia, Monroe et Cox, 2004).

\subsection{Justification du prix (plus élevé qu'un produit « conventionnel ») par les qualités intrinsèques et extrinsèques du produit du $C E$}

L'acceptation du prix passe également, dans le discours des personnes interrogées, par une justification qui repose essentiellement sur trois caractéristiques attribuées au CE.

\subsection{Bénéfices sociaux (valeur de non usage personnel)}

Les répondants estiment que le commerce équitable s'oppose au commerce conventionnel eu égard à la place accordée à l'éthique sociale : "si on décide d'acheter un produit équitable, on le fait pour une bonne action, pour soutenir certaines valeurs éthiques» (Roseline, 53 ans), «pour les produits équitables c'est le respect des critères sociaux, pour les produits ordinaires c'est des critères sanitaires » (Virginie, 27 ans). La dimension sociale a été associée surtout aux conditions de travail et à l'exploitation des enfants : "avec ces produits, on a la tranquillité d'esprit, le fait qu'on est informé que ce n'est pas un produit issu de l'esclavage », «ils ont une charte, c'est à dire qu'ils ne travaillent pas avec des enfants » (Marion, 29 ans). La question relative à la rémunération du producteur semble centrale. Elle doit permettre de subvenir durablement aux besoins des familles des producteurs : "nous savons que peu à peu, ils ont pu scolariser leurs enfants enfin, on a l'impression de faire quelque chose de positif» (Janine, 65 ans), « on travaille sur le long terme avec les producteurs » (Antoinette, 68 ans), «le revenu minimum, comme ça le producteur sait à quoi s'en tenir quand il récolte il sait "j'aurai ce prix-là" » (Marie-Laure, 66 ans). Certains situent les bénéfices à une échelle plus large, celle du territoire : «il n'y a pas que pour l'agriculteur, je pense qu'une partie doit servir pour l'aménagement, pour l'éducation, pour la formation » (Roseline, 53 ans) ou des grands équilibres sociauxdémographiques : "pour moi c'est important qu'il y ait une justice sociale. C'est un produit qui nous garantit à nous une paix sociale » (Martine, 66 ans), « c'est quelque chose de très valable [...] que les gens soient payés dans leurs pays et peuvent rester vivre dans leurs pays »(Corinne, 67 ans).

\subsubsection{Bénéfices environnementaux (valeur de non usage personnel)}

Les répondants associent par ailleurs souvent les produits équitables à un bénéfice environnemental : "le prix est juste, oui quand même ça répond aux critères de l'environnement » (Danielle, 62 ans). Ils considèrent que ces produits sont naturels : " c'est quelque que chose qui vient de la nature » (Françoise, 66 ans), et qu'ils comportent peu d'ingrédients chimiques et/ou toxiques : "dans le domaine du café équitable, il y a très peu d'insecticides, de pesticides et compagnie, les produits chimiques c'est rare » (Marie, 27 ans).

\subsubsection{Bénéfices individuels (valeur d'usage personnel)}

Les consommateurs ont par ailleurs souvent associé les produits équitables à un niveau de qualité intrinsèque élevé : «la qualité $d u$ produit, en général les produits équitables ne sont pas faits massivement, sont mieux finis » (Rémi, 41 ans), "il y a une exigence de qualité oui c'est sûr » (Marie, 27 ans). Plus particulièrement, ils ont cité le bon goût du produit et son caractère sain : "il est un petit peu plus cher mais il est très bon donc je le prends quand même » (Irène, 49 ans). 
Les discours confirment donc la convocation de mécanismes de justification chez certains répondants (Campbell, 2007), fondés notamment sur l'estimation des qualités intrinsèques et extrinsèques des produits du $\mathrm{CE}$. D'autres consommateurs justifient les prix des produits équitables par leur perception des coûts plus importants en termes de production, de certification et de logistique.

\subsection{Distribution des profits et des coûts, et réduction des inégalités dans l'échange}

Dans leurs discours, les répondants indiquent se soucier des efforts et des coûts engagés par les producteurs : " un produit ordinaire, il a le souci de l'économie, enfin le rapport $d u$ coût alors que l'équitable va se soucier plus [...] de rétribuer à sa juste valeur le travail d'une personne » (Aude, 30 ans), « un produit équitable est un produit qui respecte les dépenses engagées par le producteur $\gg$ (Jean, 26 ans). Dans ce contexte, les consommateurs considèrent qu'il est juste pour eux-mêmes de payer un peu plus pour «qu'on rémunère correctement les petits producteurs »(Sylvie, 69 ans). Il s'agit de «bonne économie » au sens d'Aristote.

Au-delà du prix payé aux petits producteurs, des répondants portent un regard sur la décomposition des prix et des marges dans la filière : "des produits équitables, c'est par rapport au prix, ce qu'on donne, la part qu'on donne au producteur, au distributeur » (Marion, 29 ans). Ils avancent des éléments d'information sur ce point : «justifié, complètement [...] pour ce qui est des Artisans du Monde ça reflète le prix au producteur, ça reflète la taxe au développement, ça reflète le transport, ça reflète la centrale d'achat et la marge des magasins » (Antoinette, 68 ans), "j'ai entendu que les producteurs doivent payer ce label Max Havelaar du coup ça leur coûte plus » (Marie-Laure, 66 ans). Ces propos semblent indiquer que les consommateurs se font une idée de la justice dans l'échange, aux différentes étapes de la filière.
Certains consommateurs mentionnent enfin la question de l'équilibre dans la relation d'échange (qui participe de l'idée de justice commutative) : «d'un point de vue humain c'est quand même important que les gens qui travaillent [...] ne soient pas écrasés par des grands acheteurs et les centrales d'achat et d'autres » (Matthieu, 32 ans). Les personnes interrogées associent donc le CE à une politique de prix qui permet de rétablir l'équilibre des pouvoirs entre les différents acteurs de la filière et de mieux distribuer les marges : "c'est lié au commerce équitable qui est gagnant-gagnant» (Claude, 41 ans). Une répondante avance même : "puisque ce sont les producteurs qui fixent ces prix, il me semble qu'on ne peut pas faire mieux » (Janine, 65 ans). Elle surestime en partie la capacité d'action des organisations du Sud dans les filières équitables mais met bien l'accent sur l'idée d'empowerment (RobertDemontrond et Ozcağlar-Toulouse, 2011). D'autres soulignent l'importance de réduire le nombre d'intermédiaires afin d'atteindre ces objectifs : " un produit équitable c'est un produit qui passe directement de la production au client et il n'y a pas trente-six intermédiaires qui engendrent plein de frais » (Hugo, 69 ans).

Au total, il y a donc chez de nombreux répondants une véritable réflexion en matière de justice commutative, conduisant à légitimer une forme de discrimination positive pour les petits producteurs du Sud, mais aussi distributive, beaucoup se prononçant sur la question de la répartition du prix dans les filières.

\subsection{Une confiance relative dans les acteurs du CE}

La confiance dans le système semble inciter certains répondants à juger que le prix des produits du CE est juste. Ce prix est, par exemple, parfois associé à une transparence concernant la part qui va au producteur, et les modes de fixation de prix : "pour le produit équitable, l'argent va vraiment au producteur »(François, 52 ans). Les consomma- 
teurs soulignent le rôle des institutions des filières équitables qui permettent d'instaurer des règles de fixation de prix et de contrôler leur applicabilité : "on a des contrôles qui permettent de vérifier que la tarification est bien faite, que toutes les étapes de la production sont correctement faites » (Marie, 27 ans). Des répondants ont d'ailleurs noté la traçabilité des produits du CE : « il y a une grosse différence, un produit ordinaire ne donne pas systématiquement le nom de l'entreprise, son origine, son producteur » (Frédérique, 28 ans).

Cependant, plusieurs expriment des doutes quant au respect des principes du CE par les acteurs du système, généralement reliés à l'impossibilité pour le consommateur de vérifier les caractéristiques réelles des produits équitables (Castaldo et alii, 2009) : " bon c'est vrai qu'on n'a pas une décomposition des prix, on n'a pas toujours très très précisément» (Antoinette, 68 ans). Des doutes sont émis sur le respect des règles : «honnêtement, je ne pense pas trop qu'ils respectent les règles »(Tristan, 24 ans), notamment concernant les prix : «il paraîtrait qu'ils perçoivent un revenu correct, il paraîtrait que oui mais est-ce que c'est la vérité ça?» (Isabelle, 50 ans), " je doute, une fois que le produit est vendu, les producteurs ne perçoivent rien selon moi »(Claude, 41 ans). Des répondants indiquent que des inégalités peuvent avoir lieu et certains acteurs du CE peuvent tirer profit au détriment des producteurs : " ah non pas de confiance, justement les critiques pour le café Malongo c'est les producteurs normalement, mais là c'est les importateurs qui ont pris » (Myriam, 36 ans).

Les doutes des répondants portent par ailleurs souvent sur les comportements des éditeurs de standards eux-mêmes et sur leurs véritables motivations. Beaucoup de critiques ont ainsi été formulées à l'encontre de Max Havelaar (Fairtrade Int), l'organisation la plus citée et la plus connue : «je crains que toute institution quelle que soit sa bonne volonté de départ, par exemple comme Max Havelaar, finisse par dévier un peu, s'habituer et c'est pour ça qu'il faut rester toujours sur ses gardes »(Janine, 65 ans). Certains consommateurs pensent que des compromis peuvent avoir lieu entre les certificateurs et la grande distribution : "là où j'ai une petite résistance, disons que c'est plutôt une question que je me pose, je ne suis pas sûre que Max Havelaar ne se soit pas compromis aussi avec la grande distribution en diminuant un peu le cahier des charges de sorte que la grande distribution y ait trouvé son compte » (Antoinette, 68 ans). Des compromis possibles entre éditeur du cahier des charges et vendeur sont donc parfois craints. Les personnes interrogées lient cette dérive à une proximité trop grande entre filières équitables et conventionnelles, en contradiction avec le principe du CE. Il s'agit notamment du développement des volumes de vente : «maintenant quand ça devient trop grand, il peut y avoir des dérives » (Viviane, 64 ans), du choix des pays et de l'existence de certains acteurs dominants dans la filière. Les répondants pointent non seulement Max Havelaar mais aussi la grande distribution : "les grandes surfaces ne savent pas appliquer des règles du travail, le code du travail que nous défendons dans les pays en développement; il y a des familles monoparentales, des femmes qui ont des horaires impossibles, des deux tiers d'emploi, [...] je ne crois pas à leur sincérité » (Janine, 65 ans). Enfin, les consommateurs évoquent la confusion engendrée par la multiplication des institutions se réclamant du CE (Diaz-Pedregal, 2007) et la pratique du greenwashing : «moi je suis assez sceptique par rapport à la responsabilité sociétale. Il y a tellement de labels on ne sait pas ce qu'il y a derrière et du coup on est assez méfiant » (Jean, 26 ans).

\section{Discussion}

Les répondants adhèrent majoritairement aux principes du $\mathrm{CE}$ et comprennent les 
valeurs additionnelles proposées par le $\mathrm{CE}$, notamment concernant les prix. Ce constat confirme d'autres travaux montrant une certaine convergence des propos au sujet du cadre d'injustice (l'ensemble des représentations partagées établissant en quoi et pourquoi une situation est injuste, cf. Le Velly, 2008) du commerce conventionnel. Dans les discours étudiés, le principe du prix juste est au cœur de leur adhésion éventuelle à cette innovation socio-économique qu'est le CE (ibid. ; Robert-Demontrond et Özçağlar-Toulouse, 2011). Mais le prix final qu'ils paient eux-mêmes n'est pas mentionné comme un élément très important. C'est aussi ce qu'avaient montré indirectement Gurviez et Sirieix (2013), lesquelles avaient expérimenté les résistances à l'achat de produits du CE en l'absence de surprix. Certes, ce résultat peut être en partie lié à un biais de désirabilité sociale, compte tenu du mode de collecte des données dans notre recherche. Mais les verbatim montrent en tous cas que les réflexions sur les prix vont bien au-delà d'une simple analyse du prix final et du prix payé au petit producteur.

La perception de la justice liée au prix se fait en effet parfois sous l'angle de la justice distributive. Les consommateurs reconnaissent toutefois ignorer ce qui se passe dans le détail en termes de prix et de marges pratiqués par les différents acteurs des filières de $\mathrm{CE}$; dès lors, ils mobilisent leurs connaissances ou leurs expériences des organisations pour se faire un avis sur les comportements effectifs. Or, c'est justement à ce niveau que se situe leur scepticisme, essentiellement en lien avec le comportement et la rémunération des acteurs centraux de la filière et en lien avec les relations entre acteurs, notamment en termes de mode de fixation des prix et des marges. Certains consommateurs sceptiques pensent que des entreprises des filières du CE peuvent se réclamer du $\mathrm{CE}$ sans en respecter les règles (Pernin et Petitpretre, 2012). Mais ces doutes viennent, davantage que d'informations qu'ils auraient à propos de tel ou tel comportement déviant, de l'idée qu'ils se font de ce que sont (ou ce que sont devenus) ces acteurs. C'est bien une dérive du type " conventionnalisation » du CE (mainstreaming en anglais, (Reynolds, 2009)) qui est perçue, c'est-à-dire un risque de perte de spécificité du CE par rapport au commerce conventionnel : soit parce que les acteurs viennent sans ambiguiité de l'univers du commerce conventionnel (les GMS) ; soit parce qu'ils deviennent trop proches de ces derniers ou « trop gros pour être honnêtes » (Fairtrade/Max Havelaar). Balineau et Dufeu (2012) avaient notamment montré la confusion possible entre éditeur du cahier des charges (Fairtrade/Max Havelaar par exemple) et vendeurs (GMS par exemple). Des consommateurs la mentionnent dans leurs propos.

Au total, la confiance/méfiance dans les institutions qui régissent le CE semble prépondérante dans la perception que les consommateurs se font de la répartition des prix et des marges. Gurviez et Sirieix (2013, p. 39) concluaient leur recherche par : « le CE semble rester une invention portée par un groupe minoritaire auquel on ne s'identifie par forcément (...) le consommateur de CE est mis à distance des autres, producteurs et promoteurs du CE, et il n'y a pas d'identification ou de sentiment d'appartenance à un groupe possible ». Les discours que nous avons étudiés permettent d'expliquer en partie ce constat. Le consommateur adhère individuellement au principe, mais ne se sent pas lié aux acteurs et ne se sent pas même en position de défendre le CE (dans sa dimension institutionnelle au moins) auprès d'autrui (discours sceptiques).

Il est possible d'en tirer quelques propositions managériales à l'endroit des acteurs des filières de CE. 


\section{Pistes de renforcement de la justice liée au prix dans le cas des produits équitables}

L'analyse des discours donne un éclairage sur les déterminants de la perception de la justice liée au prix des produits équitables. Compte tenu de ces enseignements, plusieurs recommandations stratégiques sont formulées pour permettre aux acteurs du CE de jouer favorablement sur cette perception. Le tableau 1 synthétise les objectifs que doivent viser les acteurs du $\mathrm{CE}$ et les actions à engager en conséquence.

\section{I. Renforcer la spécificité des produits équitables à travers une stratégie d'implantation adéquate}

Le jugement du prix des produits équitables comme juste, même si plus élevé que celui des produits conventionnels, est lié en partie au fait que les consommateurs comparent peu les prix des produits équitables avec les prix des produits conventionnels. Puisque les supermarchés jouent un rôle important dans la diffusion de la consommation des produits issus du CE (Diaz-Pedregal, 2007), il serait peut-être préférable qu'ils aident à renforcer l'univers particulier associé à ces produits. Dans le cas des GMS (la question se posant moins dans celui des magasins spécialisés), cette proposition renvoie à la question de l'implantation des produits du CE : doit-elle se faire dans leurs catégories de produits d'appartenance ou bien dans des linéaires dédiés aux produits du CE (De Ferran et alii, 2014) ? Les stratégies des distributeurs sont hétérogènes en la matière (comme pour les produits bio). Nous ne prétendons nullement trancher ce débat ici, mais les discours de nos répondants conduisent à privilégier la seconde option. Même s'il est tentant de positionner les produits équitables comme simples substituts aux produits conventionnels correspondants, cette tentation peut contribuer à une banalisation de ces produits et une concurrence en prix accrue avec les produits conventionnels. Outre le fait que

Tableau 1 : Comment renforcer la justice liée au prix aux yeux des consommateurs dans le cas des produits issus du CE ?

\begin{tabular}{|c|c|c|}
\hline $\begin{array}{l}\text { Déterminants de la justice liée } \\
\text { au prix }\end{array}$ & Objectif des acteurs du CE & Recommandations d'actions \\
\hline $\begin{array}{l}\text { Comparaison : faible similarité } \\
\text { avec les autres produits }\end{array}$ & $\begin{array}{l}\text { Consolider l'univers spécifique } \\
\text { des produits équitables. }\end{array}$ & $\begin{array}{l}\text { Stratégies d'implantation dans } \\
\text { les magasins (GMS) : implanta- } \\
\text { tion des produits équitables dans } \\
\text { un élément dédié. }\end{array}$ \\
\hline Justification du prix & $\begin{array}{l}\text { Rendre visible les bénéfices al- } \\
\text { truistes sans ignorer les bénéfices } \\
\text { tournés vers le consommateur. }\end{array}$ & $\begin{array}{l}\text { Mettre en avant la qualité intrin- } \\
\text { sèque des produits équitables et } \\
\text { leurs bienfaits pour le consom- } \\
\text { mateur. }\end{array}$ \\
\hline Inégalités dans l'échange & $\begin{array}{l}\text { Renforcer l'image d'une égalité } \\
\text { dans l'échange en mettant en } \\
\text { avant la place du producteur. }\end{array}$ & $\begin{array}{l}\text { Prouver la part accordée au } \\
\text { producteur : communication sur } \\
\text { le prix avec une décomposition } \\
\text { précise de la part de chaque } \\
\text { acteur. }\end{array}$ \\
\hline $\begin{array}{l}\text { Confiance relative dans les } \\
\text { acteurs du CE }\end{array}$ & $\begin{array}{l}\text { Renforcer la crédibilité des } \\
\text { acteurs et des labels du CE, tant } \\
\text { dans la rédaction que dans le } \\
\text { contrôle des cahiers des charges. }\end{array}$ & $\begin{array}{l}\text { Revoir le rôle des différents } \\
\text { acteurs dans les institutions du } \\
\text { CE et dans la distribution des } \\
\text { produits équitables : dissocier } \\
\text { entre produits du CE et marques } \\
\text { du distributeur, et limiter les } \\
\text { variations des prix. }\end{array}$ \\
\hline
\end{tabular}


cette concurrence est contraire au projet du $\mathrm{CE}$, elle fait courir le risque d'un nivellement par le bas des qualités de croyance (Balineau et Dufeu, 2012) des produits du CE.

\subsection{Convaincre les consommateurs que les produits équitables présentent aussi un bénéfice pour eux-mêmes}

Les consommateurs sont prêts à payer un supplément de prix pour un produit équitable à condition que celui-ci réponde à leurs attentes en termes de qualité intrinsèque. Tournées vers soi ou vers les autres, ces motivations sont souvent combinées et devraient être prises en compte ensemble par les acteurs de la filière. Certains acteurs du $\mathrm{CE}$ ont commencé à évoluer dans ce sens, en ne communiquant plus seulement sur les dimensions altruistes de la valeur. La première publicité télévisée sur le chocolat équitable lancée en 2014 par le pionnier français Alter $E c o$ va dans ce sens. Tout en mettant en avant le petit producteur du Sud, la marque met l'accent sur le plaisir lié à la dégustation d'un chocolat équitable.

\subsection{Prouver aux consommateurs que la part du producteur est préservée}

Plusieurs consommateurs se sont prononcés en faveur d'une justice en matière de répartition de la valeur dans les filières. Une stratégie de communication transparente sur le prix avec une décomposition précise de la part de chaque acteur semble un élément fondamental pour rendre le prix plus juste aux yeux des consommateurs. C'est ce que fait par exemple Artisans du Monde qui met à la disposition des consommateurs les informations sur la décomposition du prix du café équitable qu'elle commercialise. Dans le cas du café péruvien vendu à Artisans $d u$ Monde, la part revenant au producteur est par exemple de plus de $30 \%$, ce qui est considérablement supérieur à ce qui se pratique dans les filières conventionnelles ${ }^{7}$. Cette pratique gagnerait à se généraliser.

\subsection{Encourager la dissociation entre produits du CE et marques du distributeur, et limiter les variations des prix}

Compte tenu de la méfiance exprimée par les consommateurs concernant des acteurs historiques du CE (Fairtrade/Max Havelaar surtout), un effort accru en matière de transparence et de pédagogie est peut être nécessaire : transparence dans les négociations entre les différentes parties prenantes (Béji-Becheur, Diaz-Pedregal et ÖzçağlarToulouse, 2008), information du consommateur et pédagogie sur le contenu du cahier des charges et sur les engagements. À défaut de cette prise de conscience, Max Havelaar, peut-être trop confiant dans sa position dominante, court un risque de banalisation aux yeux des consommateurs.

Il est difficile pour les GMS et autres acteurs connus de l'univers marchand conventionnel de contrer cette méfiance liée à leur réputation. Isoler le CE de leurs autres activités est, là encore, une option possible pour les organisations conventionnelles ; c'est ce qu'a fait Danone en créant sa marque de yaourts bio «Les deux vaches » dissociée de la marque Danone, laquelle était peu liée à l'univers du bio dans l'esprit des consommateurs. Cette idée de dissociation (rayons spécifiques, marques spécifiques) pourrait être reprise pour les produits équitables des distributeurs. Le discours des répondants suggère

7/ Si on prend l'exemple du café équitable Pur Arabica provenant du Pérou qui a été commercialisé par Artisans du Monde à 4,60 € en 2012, l'information sur la décomposition du prix indique une part de $32 \%$ environ de ce prix qui revenait au producteur, une part de $22 \%$ pour la centrale d'importation et la distribution, $18 \%$ à la boutique Artisans du Monde, $7 \%$ à la Fédération Artisans du Monde qui a un rôle d'appui réseau et de représentation, $10 \%$ aux importateurs, $6 \%$ aux organisations des producteurs et enfin $2 \%$ pour la redevance Max Havelaar et le transport. 
également une autre piste pour accroître la confiance dans les produits du CE en GMS : la pratique de prix stables et homogènes dans leurs magasins. Sur le marché, souvent, les importateurs proposent des prix constants aux distributeurs, mais les prix en rayon dans chaque magasin dépendent de la stratégie de l'enseigne. Pour l'instant, ce sont davantage des négociations en amont avec les distributeurs qui sont parfois entreprises à l'instar de ce que fait Malongo ${ }^{8}$.

\section{Conclusion}

Cet article s'appuie sur des entretiens menés auprès de consommateurs français de café équitable afin d'explorer leur perception de la justice liée au prix. En nous référant à la théorie de l'équité, nous avons observé notamment que les répondants adoptent souvent des discours qui relèvent des principes de la justice distributive au sens d'Aristote. Reprenant les principes du commerce équitable à leur compte, les consommateurs se considèrent souvent explicitement comme constituant le maillon final d'une filière sur laquelle ils portent un jugement en termes de répartition des marges. Cette analyse de la façon dont les bénéfices et les coûts sont partagés entre deux parties est conforme aux principes de la théorie de la justice distributive (Homans, 1961) et de la théorie de l'équité (Adams, 1965). En lien avec ce questionnement, nous avons également constaté qu'ils considéraient la confiance qu'ils accordent aux organisations impliquées dans les filières de CE comme critique dans leur perception de la justice liée au prix, conformément aux prévisions de Ganesan (1994). Ils déclarent en revanche pratiquer peu la comparaison de prix (Xia, Monroe et Cox, 2004) au vu des spécificités de l'univers des produits équitables.

8/ Information recueillie lors d'un entretien avec le service en charge des Partenariats avec les pays producteurs.
Autrement dit, ils ont, dans leurs discours tout au moins, une vision analytique du $\mathrm{CE}$ et de la problématique des prix et des marges. Ils ne se positionnent pas directement sur le plan de la justice commutative (l'échange est-il équilibré ? Juste pour l'acheteur comme pour le vendeur ?) : ils jugent la démarche et son niveau éthique, mais ne se projettent pas réellement en tant que partenaires de l'échange et donc en tant que lésés potentiels dans la relation d'échange. Ceci peut venir $\mathrm{du}$ fait qu'ils se représentent des filières du $\mathrm{CE}$ qui sont assez longues (ils sont éloignés des petits producteurs), dans lesquelles interviennent différents acteurs dont certains sont plutôt associés au commerce conventionnel. Cette perception d'une tendance à la conventionnalisation du CE (Reynolds, 2009) est associée, dans les discours, à leur scepticisme quant à la justice des prix pratiqués dans la réalité. Malgré l'adhésion massive des répondants aux principes de la justice de prix dans le CE, cette distanciation vis-à-vis des acteurs du CE semble influencer négativement leur appropriation de cette expérience de consommation (Gurviez et Sirieix, 2013).

Ces résultats sont à considérer avec précaution, compte tenu de la méthodologie privilégiée dans ce travail et de l'échantillon mobilisé. Dans le cas du prix, d'une part, et de la consommation responsable, d'autre part, l'exercice pose sans doute un biais de désirabilité sociale. Si les répondants évitent d'aborder longuement la question du prix qu'ils ont à payer eux-mêmes, et de sa justification, n'est-ce pas en effet du fait d'une sorte de pudeur, lorsqu'ils mettent en regard leur propre situation et celle d'un petit producteur de café au Sud ? Le problème est que, lorsqu'il s'agit de passer à l'acte d'achat réel, l'arbitrage sur les prix est généralement fait par les consommateurs. Notre travail doit donc être poursuivi en adoptant une méthodologie différente permettant aux répondants de se livrer davantage quant à leurs attitudes et raisonnements intimes. En particulier, il peut être intéressant de mobiliser la méthode des 
scénarios (MDS), qui a été déjà testée dans le cadre d'études sur la dimension affective liée au prix (Dekhili, 2013), et qui a l'avantage de présenter des situations concrètes dans lesquelles les répondants peuvent se projeter pour exprimer leurs points de vue.

\section{Références}

Adams J-S. (1965), Inequity in social exchange, in L. Berkowitz (Ed.), Advances in experimental social psychology, New York : Academic Press, 2, 267-299.

Aristote (1998), Éthique à Nicomaque, Livre V (110), La justice, Ellipses.

Béji-Becheur A, Diaz-Pedregal V. et ÖzçağlarToulouse N. (2008), Fair trade - Just how « Fair » are the exchanges?, Journal of Macromarketing, 28, 1, 44-52.

Balineau G et Dufeu I. (2012), Le système Fairtrade: une garantie pour les consommateurs ?, Mondes en Développement, 4, 160, 11-26.

Campbell M-C. (2007), 'Says Who ?!' How the source of price information and affect influence perceived price (un) fairness, Journal of Marketing Research, 44, 2, 261-271.

Carrigan M. et Attalla. A (2001), The myth of the ethical consumer-do ethics matter in purchase behavior?, Journal of Consumer Marketing, 18, 7, 560-577.

Castaldo S. Perrini F. Misani N. et Tencati A. (2009), The missing link between corporate social responsibility and consumer trust: the case of fair trade products, Journal of Business Ethics, 84, $1-15$.

De Ferran F., Labbe-Pinlon B., Lombart C. et Louis D. (2014), L'implantation des produits équitables en GMS : comment favoriser l'accessibilité en magasin à ces produits responsables ?, Décisions Marketing, 75, 41-63.

Dekhili S. (2013), Les produits biologiques du point de vue du consommateur : entre le rejet de prix élevés et la méfiance envers les prix bas, Économies et Sociétés, Série « Systèmes agroalimentaires », 35, 11-12, 1859-1877.

De Pelsmacker P. Janssens W. Sterckx E. et Mielants C. (2005), Consumer preferences for the marketing of ethically labeled coffee, International Marketing Review, 22, 512-530.

Diaz-Pedregal V. (2007), Le commerce équitable dans la France contemporaine : Idéologies et pratiques, Collection Logiques Sociales, Paris, L'Harmattan.

Dufeu I, Ferrandi J.-M., Gabriel P. et Le Gall-Ely M. (2014), Multi-labellisation socio-environnementale et consentement à payer du consommateur, Recherche et Applications en Marketing, 29, 3, 34-55.

Galtier F. et Diaz Pedregal V. (2010), Le développement du commerce équitable peut-il conduire à une réduction des injustices, Cahiers Agricultures, 19, 1, 50-57.

Ganesan S. (1994), Determinants of long-term orientation in buyer-seller relationship, Journal of Marketing, 58, 2, 1-19.

Griffiths P. (2012), Ethical objections to fairtrade, Journal of Business Ethics, 105, 3, 357-373.

Gurviez P. et Sirieix L. (2013), La résistance à une innovation sociale, cadre d'analyse des problèmes de diffusion du commerce équitable, Recherche et Applications en Marketing, 28, 2, 25-45.

Homans G.C. (1961), Social behaviour: its elementary forms, New York, Harcourt, Brace \& World.

Le Velly, R. (2008). La détermination du prix équitable : Vices et vertus du prix de marché, Gestion, 33, 1, 59-65.

Martins M. et Monroe K.B. (1994), Perceived Price Fairness: A new look at an old construct, $A d$ vances in Consumer Research, 21, 1, 75-78.

Maxwell S. Nye P. et Maxwell N. (1999), Less pain, same gain: The effects of priming fairness in price negotiations, Journal of Psychology and Marketing, 16, 7, 545-562.

Olivier R.L. et Swan J.E. (1989), Consumer perceptions of interpersonal equity and satisfaction in transactions: A field survey approach, Journal of Marketing, 53, 21-35.

Pernin J-L. et Petitpretre B. (2012), Commerce équitable et scepticisme, Une typologie des consommateurs, Décisions Marketing, 66, 47-58.

PFCE (2010), Comment établir un prix équitable, Atelier professionnel, Plate-forme pour le commerce équitable, décembre, Maison des associations, Paris.

Reynolds L-T. (2009), Mainstreaming Fair Trade Coffee: From Partnership to Traceability, World Development, 37, 6, 1083-1093.

Robert-Demontrond P. (2008), Mesurer le juste prix des produits issus d'une filière « commerce équitable local » : principes, enjeux et limites épistémologiques d'une approche, Management et Avenir, 20, 6, 216-239.

Robert-Demontrond P. et Özçağlar-Toulouse N. P. (2011), Les ambiguités sémantiques du com- 
merce équitable: micro-mythanalyse des imaginaires de consommation, Recherches et Applications en Marketing, 26, 4, 53-70.

WFTO (2013), Fair Prices-Fair Wages, Workshop for WFTO Global.
Xia L. Monroe K.B. et Cox J.L. (2004), The Price is unfair! a conceptual framework of price fairness perceptions, Journal of Marketing, 68, 1-15.

\section{Annexes}

\section{Annexe I :Échantillon de consommateurs interrogés}

\begin{tabular}{|c|c|c|}
\hline \multicolumn{2}{|c|}{ Variables socio-démographiques et comportementales } & Effectif \\
\hline \multirow{2}{*}{ Sexe } & Homme & 12 \\
\hline & Femmes & 31 \\
\hline \multirow{4}{*}{ Âge } & 25 ans ou moins & 4 \\
\hline & $26-44$ ans & 16 \\
\hline & $45-65$ ans & 12 \\
\hline & Supérieur à 65 ans & 11 \\
\hline \multirow{6}{*}{ Profession } & Étudiant-doctorant & 7 \\
\hline & Employé & 3 \\
\hline & Cadre & 7 \\
\hline & Retraité & 18 \\
\hline & Demandeur d'emploi & 2 \\
\hline & Autre (commerçant, chef d'entreprise, artisan...) & 6 \\
\hline \multirow{4}{*}{ Niveau d'études } & Bac ou niveau inférieur & 9 \\
\hline & $\mathrm{Bac}+1 / \mathrm{Bac}+2$ & 11 \\
\hline & $\mathrm{Bac}+3 / \mathrm{Bac}+4$ & 8 \\
\hline & $\mathrm{Bac}+5$ et plus & 15 \\
\hline \multirow{3}{*}{$\begin{array}{l}\text { Fréquence de consom- } \\
\text { mation des produits } \\
\text { équitables }\end{array}$} & Aucune & 2 \\
\hline & Occasionnelle (moins d'une fois par mois) & 15 \\
\hline & Régulière (plus d'une fois par mois) & 26 \\
\hline \multirow{4}{*}{$\begin{array}{l}\text { Ancienneté de la } \\
\text { consommation équi- } \\
\text { table }\end{array}$} & Inférieure à 2 ans & 7 \\
\hline & $2-5$ ans & 16 \\
\hline & $6-10$ ans & 10 \\
\hline & Supérieure à 10 ans & 10 \\
\hline \multicolumn{2}{|c|}{ Nombre total des répondants } & 43 \\
\hline
\end{tabular}

\section{Annexe 2 : Guide d'entretien utilisé lors de l'étude qualitative}

\section{Parlons des produits équitables et de ce que cela évoque pour vous.}

- Selon vous, qu'est-ce qu'un produit équitable ?

- Quelles différences essentielles faites-vous entre un produit équitable et un produit ordinaire (conventionnel)?

- Faites-vous une distinction entre les produits équitables et biologiques? 
- Pensez-vous que vous êtes bien informé sur les produits équitables ? Quelles sont vos sources d'information? Faites-vous confiance à ces informations ?

- Connaissez-vous des certifications du commerce équitable ? Citez des exemples?

- Faites-vous confiance aux certifications du commerce équitable?

- Si oui, pouvez-vous préciser sur quoi porte cette confiance ?

- Si non, pour quelles raisons ?

\section{Maintenant, nous allons parler du cas du café équitable et de son prix}

- Comment jugez-vous le prix du café équitable?

- Ce prix vous semble-t-il justifié ? Qu'est-ce qu'il reflète?

- Que pensez-vous de l'idée du revenu minimum garanti pour l'agriculteur qui est derrière la notion du commerce équitable ? Pensez-vous que les producteurs perçoivent un revenu correct?

- Ce prix peut-il être un frein pour vous ?

- Que pensez-vous du café équitable vendu dans les supermarchés (et de son prix) ? est-il, selon vous, équivalent à celui vendu dans les boutiques spécialisées?

\section{Parlez-moi de vos habitudes de consommation des produits équitables ?}

- D’une façon générale, consommez-vous des produits équitables ?

- Oui, toujours

- Oui, régulièrement (plus d'une fois par mois)

- Oui, occasionnellement (moins d'une fois par mois)

- Non

Si oui, quel type de produits (café) ? Et depuis quand?

Quelles sont les raisons qui vous poussent à acheter ces produits ?

Où les achetez-vous (boutiques spécialisées, GMS, marchés...)

Si non, pourquoi ?

- Quand vous choisissez votre café équitable, sur quoi vous basez-vous pour faire ce choix? Qu'est-ce que vous regardez en premier ?

- Quel est le prix habituel que vous payez le plus souvent pour votre café équitable ? ça correspond à quelle quantité ?

- Comment trouvez-vous ce prix ?

- Y a-t-il des contextes particuliers ou occasions qui vous amènent à acheter des produits équitables (café équitable) ? Quelle sont les différentes situations (utilisation courante, un repas entre amis, achat cadeau...) ? 